\title{
An fMRI study comparing rhythmic finger tapping in children and adults
}

\author{
François De Guio, $\mathrm{PhD}^{\star}, \dagger$, Sandra W. Jacobson, $\mathrm{PhD}^{\dagger}, \S, \|$, Christopher D. Molteno, MD\|, \\ Joseph L. Jacobson, PhD ${ }^{\dagger}$, ,, , and Ernesta M. Meintjes, PhD ${ }^{*} \dagger$ \\ "MRC/UCT Medical Imaging Research Unit, Faculty of Health Sciences, University of Cape Town, \\ South Africa \\ tDepartment of Human Biology, Faculty of Health Sciences, University of Cape Town, South \\ Africa \\ $\S$ Department of Psychiatry and Behavioral Neurosciences, Wayne State University School of \\ Medicine, Detroit, MI, USA \\ IDepartment of Psychiatry and Mental Health, Faculty of Health Sciences, University of Cape \\ Town, South Africa
}

\begin{abstract}
This study compared brain activations during unpaced rhythmic finger tapping in 12-year old children with those of adults. The subject pressed a button at a pace initially indicated by a metronome (12 consecutive tones) and then continued for 16 seconds of unpaced tapping to provide an assessment of his/her ability to maintain a steady rhythm. In particular, the analyses focused on the superior vermis of the cerebellum, which is known to play a key role in timing.

12 adults and 12 children performed this rhythmic finger tapping task in a 3T scanner. Wholebrain analyses were performed in Brain Voyager with a random effects analysis of variance using the general linear model. A dedicated cerebellar atlas was used to localise cerebellar activations.

As in adults, unpaced rhythmic finger tapping in children showed activations in the primary motor cortex, premotor cortex, and cerebellum. However, overall activation was different in that adults showed much more deactivation in response to the task, particularly in the occipital and frontal cortex. The other main differences were additional recruitment of motor and premotor areas in children compared to adults along with increased activity in the vermal region of the cerebellum.

These findings suggest that the timing component of the unpaced rhythmic finger tapping task is less efficient and automatic in children, who needed to recruit the superior vermis more intensively to maintain the rhythm, even though they performed somewhat more poorly than the adults.
\end{abstract}

\footnotetext{
(c) 2011 Elsevier Inc. All rights reserved.

Corresponding Author: Ernesta M. Meintjes, Department of Human Biology, Faculty of Health Sciences, University of Cape Town, Observatory, 7925, South Africa, Tel. +27 21406 6547, Fax. +27 21448 7226, ernesta.meintjes@ gmail.com.

Publisher's Disclaimer: This is a PDF file of an unedited manuscript that has been accepted for publication. As a service to our customers we are providing this early version of the manuscript. The manuscript will undergo copyediting, typesetting, and review of the resulting proof before it is published in its final citable form. Please note that during the production process errors may be discovered which could affect the content, and all legal disclaimers that apply to the journal pertain.
} 


\section{INTRODUCTION}

Rhythmic finger tapping tasks are widely used to study neural mechanisms of timing. Rhythmicity plays an important role in performance of a broad range of cognitive and motor functions [1]. Humans are able to generate and maintain self-paced rhythmic movements and can synchronize these movements with an external rhythmic cue. In this study, a paced/ unpaced task was administered, in which the subject tapped in response to a pacing metronome and then continued tapping at the same pace without the metronome. Finger tapping tasks have often been studied in neurological disorders, such as autism spectrum disorders [2], attention-deficit/hyperactivity disorder [3], schizophrenic inpatients [4], and alcoholism [5]. Some studies have attempted to identify the brain regions associated with the complexity of rhythmic finger tapping [6]. Functional MRI (fMRI) studies of finger tapping have been widely described in the literature with some meta-analyses highlighting general findings $[7,8]$. In adults, regions involved in finger tapping have been identified within the primary sensorimotor cortices, supplementary motor area, premotor cortex, inferior parietal cortices, basal ganglia, and anterior cerebellum. Most studies of finger tapping have involved adult participants.

Neuroimaging studies have found that children often activate different or more extensive neural circuitry in performing simple tasks compared to adults. For example, in studies of number processing both adults and children activated similar neural networks, but patterns of parietal brain activation in children were significantly more extensive than in adults $[9,10]$. Konrad et al. [11] compared neural pathways associated with alerting, reorienting, and executive control of attention between children and adults and found significant differences between the two groups, i.e., children showed reduced brain activation in some regions-of-interest activated by the adults and activated a larger number of brain regions. Using a right index finger tapping paradigm, Riecker et al. [12] found that older subjects (mean age $66 \pm 8$ years) demonstrated significant overactivation within the ipsilateral sensorimotor and premotor cortex compared to young adults (mean age $23 \pm 7$ years). Agerelated group comparisons thus provide important information regarding the neural bases of cognitive development.

To our knowledge, only one fMRI study of finger tapping in healthy children has been reported [13]-a study that examined bimanual finger tapping. Because only children were assessed in that study, comparisons with adults were based on the published literature. Children activated a neural network involving primary motor cortex, supplementary motor area, and cerebellar vermis. The authors noted that children recruited more bilateral premotor areas than adults to complete the task, hence supporting a role for the presupplementary motor area (pre-SMA) in directing internally generated sequences of finger movements not specified by external cues.

This study compared brain activations during unpaced rhythmic finger tapping in 12-year old children with those found in adults. As the cerebellum plays a critical role in timing [14], a dedicated cerebellar template $[15,16]$ was used to co-register inter-subject data to improve the specificity of activations in the cerebellum, a region in which standard Talairach normalization often fails. In particular, this study focused on the superior cerebellar vermis, which is known to be important in timing processes [17]; the degree to which adults and children show deactivations during unpaced finger tapping, and the degree to which children show stronger and more extensive activations than adults to attain the same level of performance. 


\section{METHODS}

\section{Participants}

Participants were 12 right-handed adults ( 6 women), 22-29 years old (mean age $=25.0)$ and 12 right-handed children ( 7 girls), 10-13 years old (mean age $=12.0$ ). The adults were recruited from the postgraduate student body at the University of Cape Town. The children were recruited to serve as typically developing controls for neurodevelopmental studies of children with fetal alcohol spectrum disorder. All the participating children and adults were healthy with no history of neurological or psychiatric illness, and none was on medication.

\section{Procedure}

All scans were performed at the Cape Universities Brain Imaging Centre (CUBIC). A staff driver and community worker transported the mother and child from their home to CUBIC in a research-dedicated van. Written informed consent was obtained from the adult participants and the mothers of the children, who also provided oral assent. Approval for human research was obtained from the Wayne State University Human Investigation Committee and from the Faculty of Health Sciences Human Research Ethics Committee at the University of Cape Town. Mothers and children were given breakfast and a snack during the visit. The mother received a small monetary compensation, and the child was given a small gift and photo of his/her brain.

\section{Magnetic Resonance Imaging protocol}

All scans were acquired using a 3T Magnetom Allegra MRI scanner (Siemens Medical Systems, Erlangen, Germany) using the single channel head coil. High-resolution anatomical images were acquired in the sagittal plane using a three-dimensional inversion recovery gradient echo sequence $(160$ slices, $\mathrm{TR}=2300 \mathrm{~ms}, \mathrm{TE}=3.93 \mathrm{~ms}, \mathrm{TI}=1100 \mathrm{~ms}$, voxel size $=1.3 \times 1.0 \times 1.0 \mathrm{~mm}^{3}$, scan time $=6: 03$ minutes). During the fMRI protocol, 120 volumes sensitive to blood oxygen level dependent (BOLD) contrast were acquired with a T2*-weighted gradient echo, echo planar imaging sequence $(\mathrm{TR}=2000 \mathrm{~ms}, \mathrm{TE}=30 \mathrm{~ms}, 34$ interleaved slices, $3 \mathrm{~mm}$ thick, $0.9 \mathrm{~mm}$ gap, $200 \times 200 \mathrm{~mm}^{2}$ field of view, matrix size $64 \times$ 64 , resolution $3.125 \times 3.125 \times 3 \mathrm{~mm}^{3}$ )

\section{Functional MRI task}

All subjects completed a rhythmic finger tapping task, which they practiced before being scanned. This task is easy enough to be performed correctly by children, permitting a comparison of activations with those found in adults. The children also listened to a recording of the scanner noises while lying in a mock scanner prior to scanning, which was important in reducing anxiety and facilitating completion of the MRI scans. The experimental task was programmed using E-Prime software (Psychology Software Tools, Inc., Pittsburgh, USA) and was presented using a data projector positioned in line with the bore of the magnet in the control room behind the scanner. Images were projected through a waveguide onto a rear projection screen mounted behind the scanner, which subjects viewed using the standard mirror system that mounts to the head coil. Auditory tones were presented using the standard Siemens headphones. Subjects held a four-button Lumitouch response system (Photon Control Inc., Burnaby, Canada) in their right hand and responded using their right index-finger. Button presses were recorded on the stimulus presentation computer. Subjects were able to talk to the examiner using an intercom that is built into the scanner and could stop the scan at any time by squeezing a ball held in their left hand. All children were accompanied by a research nurse or research assistant whom they knew from previous assessments, who sat near the scanner throughout the scan, which also contributed to the high success rate of the scanning sessions. 
The rhythmic tapping task, administered using a block design, consisted of alternating blocks of rest, paced tapping, and unpaced tapping. In the paced tapping blocks ( 6 seconds duration), subjects were presented with a pacing metronome while viewing a blank black screen. The pacing metronome comprised 12 auditory $1 \mathrm{kHz}$ tones, presented every 500ms. Subjects were instructed to press the button with their right index finger every time they heard a beep. Unpaced blocks were 16 seconds in duration. Subjects viewed a blank black screen while continuing to tap at the same pace as the pacing metronome that was presented during the paced block. During the rest block, the instruction 'Stop' was displayed for 12 seconds, followed by the words 'Get Ready ...', which were displayed for 4 seconds. All blocks were repeated 6 times. The final rest block consisted only of the word 'Stop' presented for 16 seconds. The task was triggered to begin automatically with the start of the acquisition of the 5th EPI BOLD volume, after which the instruction 'Get Ready ...' was initially displayed for 4 seconds prior to the onset of the first paced tapping block. All button presses were recorded on a computer. For one adult an incorrect version of the task was run, which only had four repeats of each block.

Unpaced blocks were classified as either good or bad based on subject performance. Blocks were labelled as bad if the mean inter-tap interval was less than $400 \mathrm{~ms}$ or greater than 600 $\mathrm{ms}$, if the standard deviation of the mean inter-tap interval was greater than $65 \mathrm{~ms}$, or if the subject missed more than 5 taps within a block. We assumed that a subject had missed a tap if the inter-tap interval was greater than $800 \mathrm{~ms}$. When that occurred, the inter-tap interval was divided by 2 for the computation of the mean, and 1 missed tap was counted. Subjects who did not complete at least 3 unpaced blocks successfully were excluded from the analysis. Based on these criteria, two children were excluded due to a too rapid tapping rate (mean of inter-tap time $<400 \mathrm{~ms}$ ) in 3 unpaced blocks. We thus present data for 10 children and 12 adults. Of the data included in the analysis, 6 of 60 and 5 of 70 unpaced blocks were considered 'bad' due to too many missed taps or excessive standard deviation for the children and adults, respectively.

\section{5. fMRI data analysis}

Brain Voyager QX (Brain Innovation, Maastricht, The Netherlands) and SPM5 (http://www.fil.ion.ucl.ac.uk/spm) were used to analyze the fMRI data. Brain Voyager was used to perform all pre-processing, whole-brain co-registration, Talairach normalization, and whole-brain analyses. Four dummy images were acquired in each run that were excluded from all analyses. Preprocessing included correction for different slice acquisition times and linear trends, and temporal smoothing with a high pass filter of 3 cycles/point. Images were motion corrected relative to the first volume of the first functional run within the scanning session with trilinear estimation and interpolation. All data exceeding movement criteria of 3 $\mathrm{mm}$ displacement or $3.0^{\circ}$ rotation within a functional run were rejected.

Each functional data set was co-registered to its corresponding high-resolution iso-voxeled anatomical image, rotated into the anterior commissure-posterior commissure (AC-PC) plane and normalized to the standardized Talairach space using a linear transform calculated on the anatomical images. The $3.125 \times 3.125 \times 3 \mathrm{~mm}^{3}$ fMRI voxels were interpolated during Talairach normalization to $3 \times 3 \times 3 \mathrm{~mm}^{3}$.

Whole-brain analyses were performed in Brain Voyager with a random effect analysis of variance using the general linear model with predictors based on the known experimental blocks (Paced, Unpaced, Unpaced_Bad) convolved by the standard hemodynamic function. The six motion correction parameters were added as predictors of no interest. Beta maps were created for each subject for the contrast of interest (Unpaced > Rest) and analysed at the second level using an analysis of variance (ANOVA) approach with one within-subjects and one between-subjects factor. 
In order to improve the specificity of the activations in the cerebellum where co-registration and inter-subject alignment accuracy are often poor using Talairach normalization, we repeated the $2^{\text {nd }}$ level analysis using the spatially unbiased cerebellar atlas template (SUIT) for the cerebellum developed by Diedrichsen $[15,16]$. We exported both the high resolution Talairach-normalized anatomical images and the beta maps for the Unpaced > Rest contrast from Brain Voyager to SPM5 for each subject. Both these volumes were rotated and normalized to Montreal Neurological Institute (MNI) space. Using functions from the SUIT package, we isolated the cerebellum in the anatomical images, normalized the segmented cerebellum to the SUIT template, and applied the same geometrical transformation to the functional data. Once the beta maps of each subject had been co-registered to the SUIT template, the $2^{\text {nd }}$ level analysis was run within SPM5 using an ANOVA approach with two groups. Finally, the probabilistic atlas in SUIT space was used to assign labels to different cerebellar activations in an unbiased fashion.

\section{RESULTS}

\section{Behavioural results}

Mean and standard deviation (SD) of inter-tap intervals for adults and children for all blocks (good and bad) are summarized in Table 1. For each group, mean inter-tap time during selfpaced tapping is close to the metronome interval $(500 \mathrm{~ms})$, and standard deviation is much less than $65 \mathrm{~ms}$. Adults performed somewhat better as they maintained a more regular rhythm ( $\mathrm{SD}=28.1 \mathrm{~ms}$ ) compared to children $(\mathrm{SD}=41.9 \mathrm{~ms})(\mathrm{p}<0.07)$. These results are in agreement with those found in the literature. For example, SD was $23.6 \mathrm{~ms}$ for the unpaced tapping condition in adult controls in the rhythmic finger tapping task in Ivry and Keele [14], and for the $2 \mathrm{~Hz}$ finger tapping task in Corriveau et al. [18] it was $55 \mathrm{~ms}$ for control children ( 9.8 years mean age).

\section{Neuroimaging results}

a) Adults-The cortical activations in adults for the simple contrast Unpaced tapping > Rest were unexpected. At $p_{\text {uncorrected }}<0.0001$, the whole brain analysis yielded deactivations only, especially in occipital and frontal regions, i.e., these regions showed significantly less activity during the tapping blocks compared to the rest periods. Only at a much more relaxed threshold of $\mathrm{p}_{\text {uncorrected }}<0.05$, a positive activation in the left central sulcus was observed. By contrast, in the cerebellum using the SUIT normalization, the regions classically associated with motor tasks were found activated at $\mathrm{p}_{\text {uncorrected }}<0.01$; namely 1 st motor representation in the anterior ipsilateral region and superior and inferior vermis, and 2nd motor representation in lobule VIII.

b) Children-Brain regions showing either significantly more or less activity during unpaced tapping compared to rest in children are listed in Table 2. The children showed increased activity during unpaced tapping compared to rest in the left paracentral lobule close to the supplementary motor area, corresponding to Brodmann's area (BA) 6, which forms part of the premotor cortex. The largest area of activation in children was the left precentral gyrus including BA1, BA2, BA4, and BA5, representing primary motor cortex and secondary somatosensory cortex. Left thalamus and claustrum were also significantly activated during the task. Several areas were active in the visual cortex (BA17, BA18, BA19). In the cerebellum (Fig. 1), two large areas were activated: the right ipsilateral cerebellum (lobules IV, V and VI), extending from the superior vermis with high peak values close to the midline to more lateral regions (1st motor representation), and a posterior region close to the midline (Vermis_VIIIa, Right_VIIIa, Vermis_VIIb, Right_IX). Other activated regions included bilateral Crus II, Right_VIIb and Left_VIIIa. 
c) Children > Adults-Table 3 lists regions where the activity in children and adults differed significantly. With a strict threshold, there were no regions in the whole-brain analysis where adults showed more activity than children. Children showed more activity than adults (Table 3) in bilateral motor (precentral gyrus, paracentral lobule (BA4)), left premotor (superior frontal gyrus (BA6)), and left somatosensory (BA1, BA2, BA5) areas during unpaced tapping. The highest $t$ value was seen in the largest area, which was the left precentral gyrus. There were also some differences in activation in the right thalamus, the middle temporal gyrus (BA21) and several spots in the occipital lobe (BA17, BA19). In the cerebellum, the superior vermis (lobule $\mathrm{V}$ around the midline), the inferior vermis, bilateral crus II, lobules right VIIb, and left VIIIa were activated more in the children than in the adults. All these regions were significantly activated in the children.

\section{DISCUSSION}

Several finger tapping studies in adults report fMRI activation of the contralateral sensorimotor cortex, ipsilateral cerebellum and additional activation of areas, such as the basal ganglia, thalamus, and sensory cortices during uni-manual hand movement compared with rest $[6,7,19,20]$. In this study, we found with a relaxed threshold that left primary motor cortex, left somatosensory cortex, and left SMA were activated in the adult group. In their meta-analysis, Witt et al. [7] also found that for auditory paced tasks, the left ventral premotor cortex was activated. In the cerebellum, the first of the two main areas (lobules IV, $\mathrm{V}, \mathrm{VI}$ ) that were activated is classically known as the first motor representation or the hand area (as depicted in Fig. 1) [21-23]. Areas in motor cortex and in the motor part of the cerebellum are connected and form independent modular loops [24]. The other area in the cerebellum that was activated (lobules VII, VIIIa, VIIIb) has emerged as the second cluster in meta-analyses of sensorimotor studies and is known as the secondary representation of hand movements [21-23].

A strong deactivation was found in adults in this study. Indeed, for a high threshold such as $\mathrm{p}_{\text {uncorrected }}<0.0001$, we observed only regions of deactivation. Only on decreasing the threshold to $\mathrm{p}_{\text {uncorrected }}<0.05$, did we observe activation in the primary motor cortex. One possible explanation for this deactivation could be that hand movements are associated with contralateral cerebral activation and ipsilateral cerebral deactivation (decreased blood flow) as shown in Allison et al. [25]. This could explain the right sensorimotor deactivation. Deactivations in the occipital lobe could be due to cross-modal influences. In the presence of one modality-specific stimulus, synaptic activity in the cortex of a nonmatching sensory modality can be reduced. Thus, superior and middle occipital gyri, which are involved in various aspects of visual processing, will be strongly deactivated in response to auditory stimuli [26]. Previous studies have demonstrated that somatosensory stimulation can also produce deactivation of the visual cortex [27]. Similarly, negative activation during visuallycued finger tapping has been found in auditory-related areas by Kudo et al. [28]. The children did not exhibit the extensive deactivations in the occipital lobe that were seen in the adults.

Similar to our results, activations in the contralateral primary sensorimotor cortex and the right ipsilateral cerebellum during finger tapping have been reported for control children (mean age $=10.4$ years) by Mostofsky et al. [3]. Mostofsky et al. [2] also demonstrated activation in the contralateral pre/postcentral gyrus, ipsilateral cerebellum (lobules IV/V), bilateral superior medial wall (BA6), and contralateral thalamus, in typically developing children (mean age $=10.9$ years old) when tapping each finger to the thumb. Riecker et al. [29] reported activation of the left thalamus during finger tapping, which is one of the regions activated by the children in our study (Table 2). 
Bilateral premotor activation has been reported in Rivkin et al. [13] in a bimanual finger tapping study in children as well as anterodorsomedial cerebellum activation during self tapping that corresponds to the vermal activation found in this study. These authors also hypothesized a role for the pre-SMA (anterior to the perpendicular intersector of the line between the anterior and posterior commisures and passing through the anterior comissure) in directing internally generated sequences of finger movements not specified by external cues in children, since this was not observed in adults. Unlike Rivkin et al. [13], midline motor activation was only found posterior (SMA) to the perpendicular intersector in this study. Overall, children showed greater activation than adults, with no regions showing greater activation during unpaced tapping in adults, even for a very relaxed threshold. Although it has been shown that BOLD signal increases with age in childhood from 5 to 19 years for several tasks including paced tapping [30], it seems that for this task where an internal metronome needs to be maintained, children exert more mental effort. Considering that both groups showed a nearly similar pattern of activation in the brain and the cerebellum (with a difference in signal intensity) and that both groups performed well on the task, we can reasonably conclude that the observed differences represent a difference in the neural organization that mediates unpaced rhythmic finger tapping according to an internal previously learnt metronome.

The ANOVA approach with a direct group comparison enabled the localization of differences in activation. While both adults and children activated the left motor area, children showed greater activation of primary motor cortex (L precentral gyrus). This greater activation was observed bilaterally but was stronger on the left side in terms of tvalue and cluster size. Children also showed greater activation in left premotor areas and in the somatosensory cortex. Traditionally, the premotor cortex was linked to the execution of simple movements under sensory guidance [31]. In adults, the premotor cortex has been shown to play an important role in the transformation of sensory information into appropriate motor behaviour especially in regards to sequential movements [32]. While premotor was definitely more active in children than in adults, we did not observe increased pre-SMA activation in the children during self-paced tapping as found by Rivkin et al. [13], possibly because this task is motorically much less complex than the bimanual finger tapping task they used.

In the cerebellum, using the SUIT template, the strongest $t$ value and largest cluster for the contrast Children > Adults was the right Crus II (Fig. 1). Bilateral Crus II was activated in children only. Crus II is known to be involved in many cognitive tasks and forms part of the executive network with the prefrontal cortex and parietal cortex [23,33]. Ramnani [34] has predicted that during the acquisition of cognitive skills that become increasingly stereotyped and automatic, a decline in prefrontal activity will be accompanied by increasing activity in connected areas (including Crus II).

Activation of the secondary motor representation (Vermis_VIIb, Vermis_VIIIa) in the cerebellum was also greater in children than in adults, suggesting that overall children need to recruit more motor and premotor resources than adults to perform the self-paced finger tapping task.

Finally, children showed greater activation of the superior vermis than adults (MNI coordinates $[4-61-21]$ with tmax $=4.4$, cf Table 3 and Fig. 1). This region corresponds to the vermal part of the large cluster identified as 1 st motor representation and superior vermis in the children (MNI coordinates [3-61 -21] with tmax $=8.2$, cf Table 2). In fact, both groups activated the classical hand area (lateral lobules IV, V, VI), but children activated more of the superior vermis. This region is believed to be involved in rhythmic tapping and is specifically important in discrete movements [17]. Spencer et al. [17] found a peak 
activation for discrete movements at coordinates [0 -62-20], which is close to the region activated in children in our study and an area where children show greater activity than adults, suggesting that children show more activity specifically in this cerebellar region that has been implicated in timing.

Given that cerebellar loci in motor regions form neural circuits with both primary motor cortex and supplementary motor area, it is not surprising to find an overactivation in children compared to adults in all these linked areas. Midline cerebellar structures also project via thalamic nuclei $[6,13]$ (children showed greater activity in the right thalamus) not only to motor and premotor cortex but also to SMA, prefrontal, posterior parietal and temporal regions [13], which is consistent with the greater activation observed in children in frontal and temporal regions in Table 3.

Another region that was significantly more activated in children than adults during unpaced finger tapping, was the left middle temporal gyrus (BA21). Auditory cortices were activated for both groups during paced tapping when subjects listened to the auditory metronome. But when the metronome stopped, children continued to activate the auditory cortex, suggesting that they either try to continue hearing the beeps or perhaps imagine the beeps to assist them in tapping without the metronome.

Comparing functional data between two groups with different ages raises questions about the appropriate template/atlas to use. As the focus of this study was a direct comparison, we chose to use classic Talairach normalization for the whole-brain analysis and SUIT normalization for the cerebellum. Hence, the smaller brains of the children have been aligned to a standard adult brain regardless of their morphology. Nevertheless, given the spatial resolution and the spatial filtering of fMRI volumes, it has been shown that differences in anatomy between the brains of 8-year-old children and adults do not affect functional imaging data substantially when compared within the same stereotactic space [35]. Moreover, looking at each group's activation, networks involved in finger tapping were in agreement with those reported in the literature. In the cerebellum, the SUIT normalization yields a better co-registration between subjects and from our results has proven to be useful in studies of children as well.

\section{CONCLUSION}

As in adults, rhythmic finger tapping in children involves brain activations in the primary motor cortex, premotor cortex, and cerebellum. However, overall activation is different as adults show much more deactivation in response to the task, presumably due to cross-modal influences, showing the need for age-matched controls in fMRI studies dealing with motor tasks. The main difference entails the additional recruitment of motor and premotor areas (in the frontal cortex and in the cerebellum) in children compared to adults. The use of the SUIT template and associated functions to isolate and normalize the cerebellum better highlights these age differences. Indeed, the nonlinear normalization of the cerebellum enabled a more accurate inter-subject-alignment leading to activated regions which were more localized and more significant than those initially found with the Talairach normalization. The two clusters classically associated with hand movements were activated in children. Children show greater activity in the secondary representation of motor, in Crus II, and in the superior vermis (lobule $\mathrm{V}$ ), which is involved in discrete movements and timing. These findings suggest that the timing component of the unpaced finger tapping task is less automatic in children as they needed to recruit the superior vermis more to maintain the rhythm, even while performing somewhat more poorly than adults. 


\section{Acknowledgments}

Funding: Supported by grants from the National Research Foundation of South Africa (FA2005040800024), NIH Fogarty Foundation (R03 TW007030), the South African Research Chairs Initiative of the Department of Science and Technology and the National Research Foundation, and seed money grants from the Office of the President, Wayne State University, and the Joseph Young, Sr., Fund of the State of Michigan.

We thank Mariska Pienaar, Maggie September, and Katie Bromley, University of Cape Town, for their contributions to the data collection, radiographers Marie-Louise de Villiers and Fran Dawson, the staff at CUBIC, and Siemens Medical Solutions-Southern Africa. We also thank Richard Ivry, PhD, University of California, Berkeley, for his generous input in the development of the task, and Richard Ivry and John Schlerf, PhD, for their input in the interpretation of the data.

\section{References}

1. Thaut MH, Stephan KM, Wunderlich G, Schicks W, Tellman L, Herzog H, McIntosh GC, Seitz RJ, Hömberg V. Distinct cortico-cerebellar activations in rhythmic auditory motor synchronization. Cortex. 2009; 45:44-53. [PubMed: 19081087]

2. Mostofsky SH, Powell SK, Simmonds DJ, Goldberg MC, Caffo B, Pekar JJ. Decreased connectivity and cerebellar activity in autism during motor task performance. Brain. 2009; 132:2413-25. [PubMed: 19389870]

3. Mostofsky SH, Rimrodt SL, Schafer JGB, Boyce A, Goldberg MC, Pekar JJ, Denckla MB. Atypical Motor and Sensory Cortex Activation in Attention-Deficit/Hyperactivity Disorder: A Functional Magnetic Resonance Imaging Study of Simple Sequential Finger Tapping. Biol Psychiatry. 2006; 56:48-56. [PubMed: 16139806]

4. Muller JL, Roder CH, Schuierer G, Klein H. Motor-induced brain activation in cortical, subcortical and cerebellar regions in schizophrenic inpatients. A whole brain fMRI fingertapping study. Prog Neuropsychopharmacol Biol. 2002; 26:421-6.

5. Parks MH, Morgan VL, Pickens DR, Price RR, Dietrich MS, Nickel MK, Martin PR. Brain fMRI Activation Associated with Self-Paced Finger Tapping in Chronic Alcohol-Dependent Patients. Alcohol Clin Exp Res. 2003; 27:704-11. [PubMed: 12711934]

6. Dhamala M, Pagnoni G, Wiesenfeld K, Zink CF, Martin M, Berns GS. Neural correlates of the complexity of rhythmic finger tapping. Neuroimage. 2003; 20:918-26. [PubMed: 14568462]

7. Witt ST, Laird AR, Meyerand ME. Functional neuroimaging correlates of finger-tapping task variations: An ALE meta-analysis. Neuroimage. 2008; 42:343-56. [PubMed: 18511305]

8. Chan RCK, Huang J, Di X. Dexterous movement complexity and cerebellar activation: A metaanalysis. Brain Res Brain Res Rev. 2009; 59:316-23.

9. Davis N, Cannistraci CJ, Rogers BP, Gatenby JC, Fuchs LS, Anderson AW, Gore JC. The neural correlates of calculation ability in children: an fMRI study. Magn Reson Imaging. 2009; 27:118797. [PubMed: 19570639]

10. Meintjes EM, Jacobson SW, Molteno CD, Gatenby JC, Warton C, Cannistraci CJ, Gore JC, Jacobson JL. An fMRI study of magnitude comparison and exact addition in children. Magn Reson Imaging. 2010; 28:351-62. [PubMed: 20116955]

11. Konrad K, Neufang S, Thiel CM, Specht K, Hanisch C, Fan J, Herpertz-Dahlmann B, Fink GR. Development of attentional networks: An fMRI study with children and adults. Neuroimage. 2005; 28:429-39. [PubMed: 16122945]

12. Riecker A, Groschel K, Ackermann H, Steinbrinck C, Witte O, Kastrup A. Functional significance of age-related differences in motor activation patterns. Neuroimage. 2006; 32:1345-54. [PubMed: 16798017]

13. Rivkin MJ, Vajapeyam S, Hutton C, Weiler ML, Hall EK, Wolraich DA, Yoo SS, Mulkern RV, Forbes PW, Wolff PH, Waber DP. A functional magnetic resonance imaging study of paced finger tapping in children. Pediatr Neurol. 2003; 28:89-95. [PubMed: 12699857]

14. Ivry RB, Keele SW. Timing functions of the cerebellum. J Cogn Neurosci. 1989; 1:136-52.

15. Diedrichsen J. A spatially unbiased atlas template of the human cerebellum. Neuroimage. 2006; 33:127-38. [PubMed: 16904911] 
16. Diedrichsen J, Balsters JH, Flavell J, Cussans E, Ramnani N. A probabilistic MR atlas of the human cerebellum. Neuroimage. 2009; 46:39-46. [PubMed: 19457380]

17. Spencer RMC, Verstynen T, Brett M, Ivry RB. Cerebellar activation during discrete and not continuous timed movements: An fMRI study. Neuroimage. 2007; 36:378-87. [PubMed: 17459731]

18. Corriveau KH, Goswami U. Rhythmic motor entrainment in children with speech and language impairments: Tapping to the beat. Cortex. 2009:119-130. [PubMed: 19046744]

19. Jancke L, Loose R, Lutz K, Specht K, Shah NJ. Cortical activations during paced finger-tapping applying visual and auditory pacing stimuli. Cognit Brain Res. 2000; 10:51-66.

20. Lewis PA, Wing AM, Pope PA, Praamstra P, Miall RC. Brain activity correlates differentially with increasing temporal complexity of rhythms during initialisation, synchronisation, and continuation phases of paced finger tapping. Neuropsychologia. 2004; 42:1301-12. [PubMed: 15193939]

21. Desmond JE, Gabrieli JDE, Wagner AD, Ginier BL, Glover GH. Lobular patterns of cerebellar activation in verbal working-memory and finger-tapping tasks as revealed by functional MRI. $\mathrm{J}$ Neurosci. 1997; 17:9675-85. [PubMed: 9391022]

22. Grodd W, Hulsmann E, Lotze M, Wildgruber D, Erb M. Sensorimotor mapping of the human cerebellum: fMRI evidence of somatotopic organization. Hum Brain Mapp. 2001; 13:55-73. [PubMed: 11346886]

23. Stoodley CJ, Schmahmann JD. Functional topography in the human cerebellum: A meta-analysis of neuroimaging studies. Neuroimage. 2009; 44:489-501. [PubMed: 18835452]

24. Hayter AL, Langdon DW, Ramnani N. Cerebellar contributions to working memory. Neuroimage. 2007; 36:943-54. [PubMed: 17468013]

25. Allison JD, Meador KJ, Loring DW, Figueroa RE, Wright JC. Functional MRI cerebral activation and deactivation during finger movement. Neurology. 2000; 54:135-142. [PubMed: 10636139]

26. Laurienti PJ, Burdette JH, Wallace MT, Yen YF, Field AS, Stein BE. Deactivation of SensorySpecific Cortex by Cross-Modal Stimuli. J Cogn Neurosci. 2006; 14:420-9. [PubMed: 11970801]

27. Macaluso E, Frith CD, Driver J. Modulation of human visual cortex by crossmodal spatial attention. Science. 2000; 289:1206-8. [PubMed: 10947990]

28. Kudo K, Miyazaki M, Kimura T, Yamanaka K, Kadota H, Hirashima M, Nakajima Y, Nakazawa $\mathrm{K}$, Ohtsuki T. Selective activation and deactivation of the human brain structures between speeded and precisely timed tapping responses to identical visual stimulus: an fMRI study. Neuroimage. 2004; 22:1291-301. [PubMed: 15219601]

29. Riecker A, Wildgruber D, Mathiak K, Grodd W, Ackermann H. Parametric analysis of ratedependent hemodynamic response functions of cortical and subcortical brain structures during auditorily cued finger tapping: a fMRI study. Neuroimage. 2003; 18:731-9. [PubMed: 12667850]

30. Schapiro MB, Schmithorst VJ, Wilke M, Byars AW, Strawsburg RH, Holland SK. BOLD fMRI signal increases with age in selected brain regions in children. Neuroreport. 2004; 17:2575-8. [PubMed: 15570155]

31. Goldberg G. Supplementary motor area structure and function: review and hypotheses. Behav Brain Sci. 1985; 8:567-616.

32. Harrington DL, Rao SM, Haaland KY, Bobholz JA, Mayer AR, Binderx JR, Cox RW. Specialized neural systems underlying representations of sequential movements. J Cogn Neurosci. 2000; 12:56-77. [PubMed: 10769306]

33. Habas C, Kamdar N, Nguyen D, Prater K, Beckmann CF, Menon V, Greicius MD. Distinct cerebellar contributions to intrinsic connectivity networks. J Neurosci. 2009; 29(26):8586-94. [PubMed: 19571149]

34. Ramnani N. The primate cortico-cerebellar system: anatomy and function. Nat Rev Neurosci. 2006; 7:511-522. [PubMed: 16791141]

35. Burgund ED, Kang HC, Kelly JE, Buckner RL, Snyder AZ, Petersen SE, Schlaggar BL. The feasibility of a common stereotactic space for children and adults in fMRI studies of development. Neuroimage. 2002; 17:184-200. [PubMed: 12482076] 


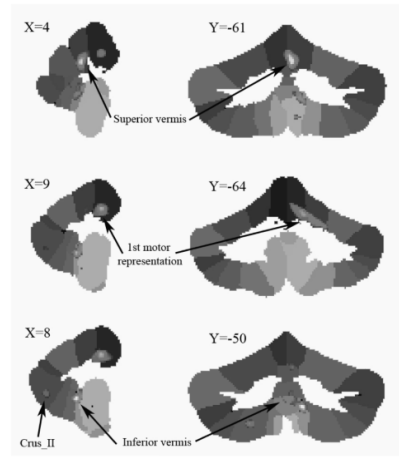

Figure 1.

Activation for children in cerebellum SUIT space (MNI coordinates) for the Unpaced tapping > Rest contrast, showing $1^{\text {st }}$ and $2^{\text {nd }}$ motor representation, superior and inferior vermis, and bilateral Crus II. 
Table 1

Task performance for adults and children

\begin{tabular}{lcc}
\hline & Adults & Children \\
Mean - paced tapping (ms) & 532.4 & 531.8 \\
Mean - unpaced tapping (ms) & 515.2 & 514.2 \\
Standard deviation - unpaced tapping (ms) & 28.1 & 41.9 \\
\hline
\end{tabular}




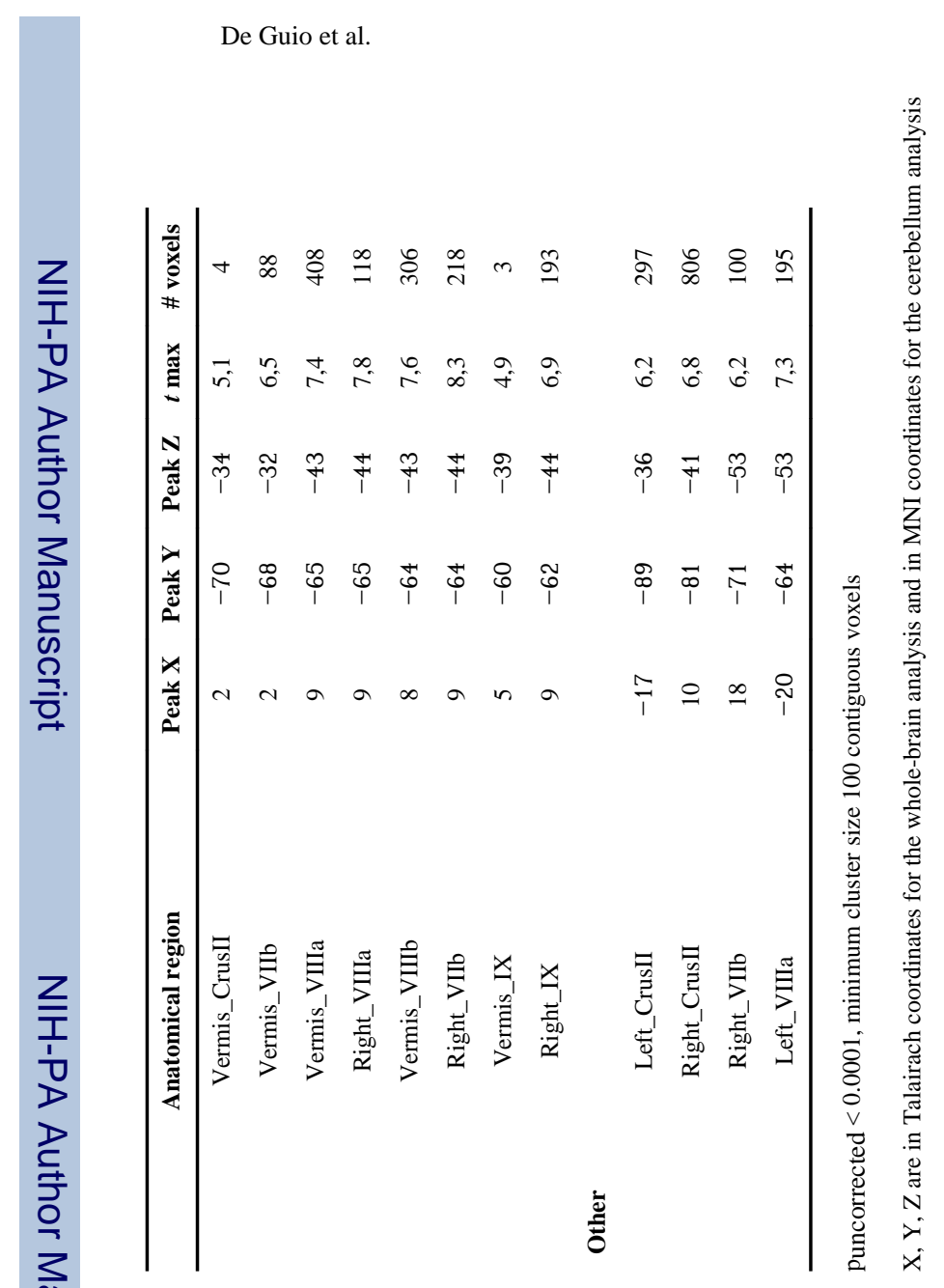

Page 14 


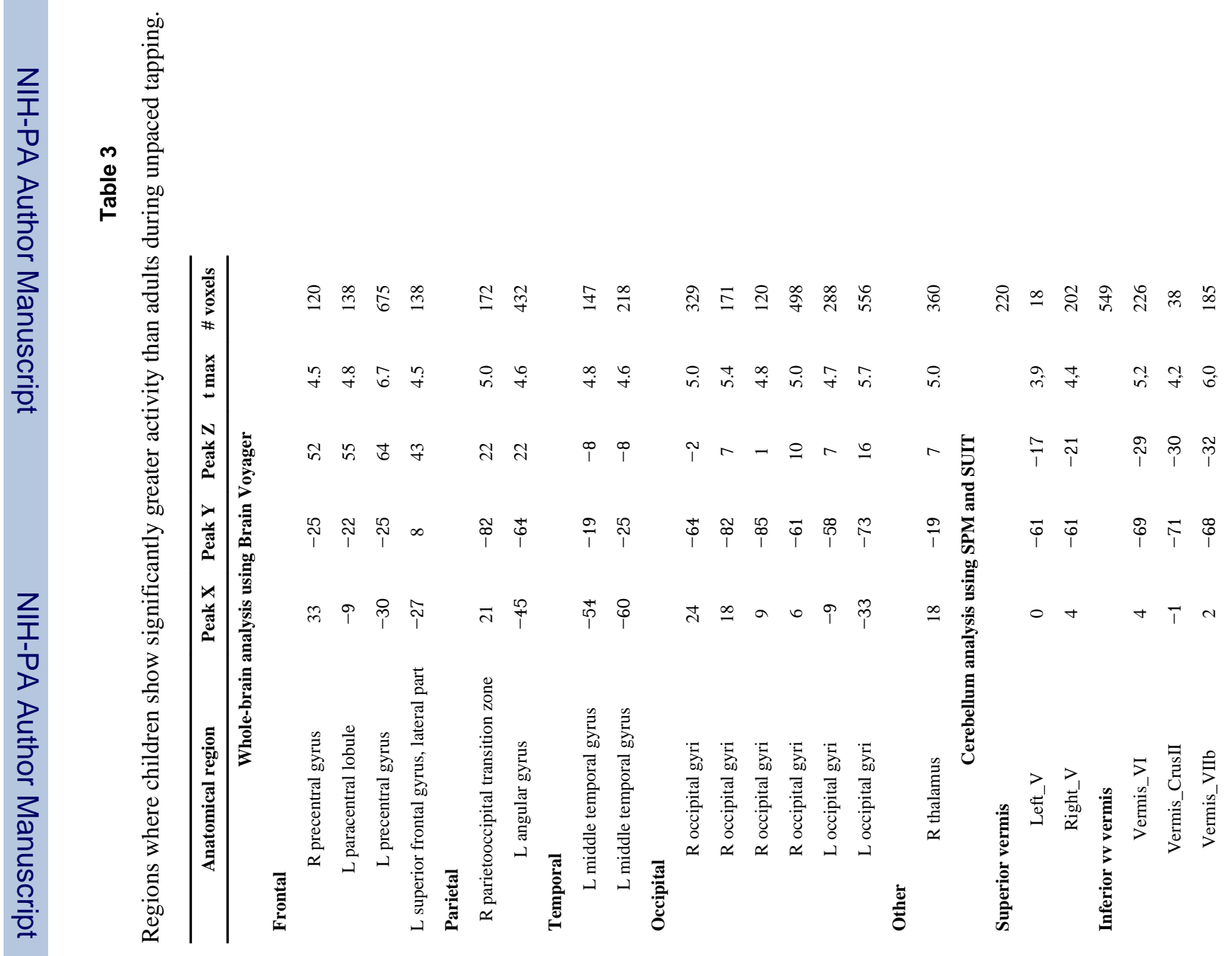
Pediatr Neurol. Author manuscript; available in PMC 2013 February 1. 


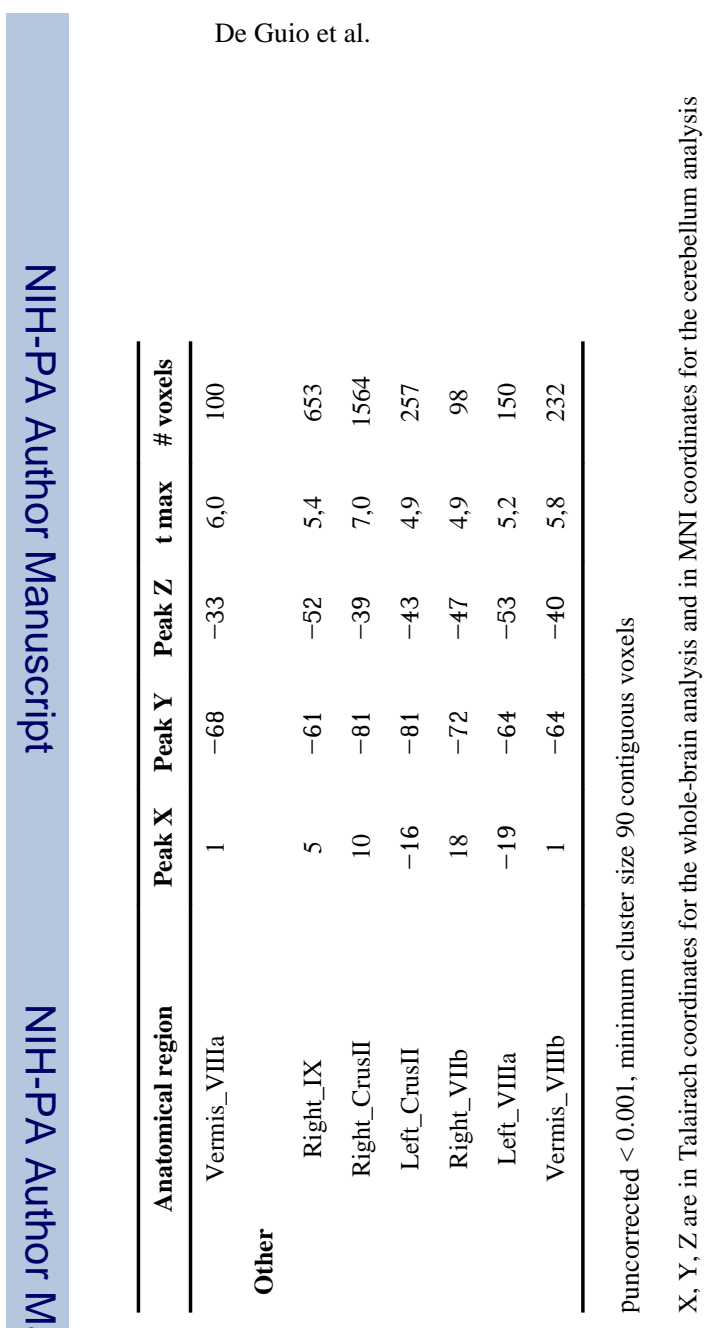

Page 16

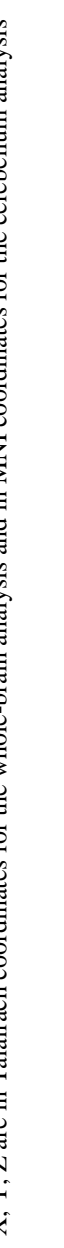

(1)

